\title{
Stress model for the wrinkling of ion-implanted layers
}

\author{
C. Hajdu, F. Pászti, I. Lovas, and M. Fried \\ Central Research Institute for Physics of the Hungarian Academy of Sciences, P.O. Box 49, Budapest, H-1525 Hungary
}

(Received 20 July 1989)

\begin{abstract}
A model is presented to describe the regular ripple patterns observed in several kinds of high-dose ion-implantation experiments. The model is based on elastic instability of the implanted layer, occurring when a critical value of the lateral stress is reached during ion implantation. The predicted wavelength of wrinkling is related to the width of the implanted-atom distribution, and it is in good agreement with experimental results.
\end{abstract}

\section{INTRODUCTION}

Fine periodic structures have for a long time been observed on sputtering eroded surfaces, ${ }^{1}$ and more recently, similar features were also found on various targets after blistering, flaking, or exfoliation induced by ions in the $\mathrm{MeV}$ energy range. ${ }^{2-5}$ The evolution of these ripple patterns is not understood, although several explanations have been proposed. ${ }^{1,6-8}$ Our model is based on the elastic instability of the implanted layer occurring when a critical value of the lateral stress is reached during ion implantation. Depending on experimental conditions, the ripple structure induced by elastic instability is either observable directly on the sample surface, or it may only be made visible after other surface deformation processes take place.

\section{THE MODEL}

To model an ion-implanted sample, let us consider a homogeneous, isotropic semispace with its uppermost layer of thickness $h$ subjected to uniform stress parallel to the surface lying in the $x-y$ plane. Let the unstressed part of the semispace exert a restoring force on the stressed plate proportional to its perpendicular deflection. Equilibrium of this thin plate of thickness $h$ is described by the following set of equations: ${ }^{9}$

$$
\begin{gathered}
D \Delta^{2} W-h\left[\frac{\partial}{\partial x}\left[\sigma_{x x} \frac{\partial W}{\partial x}+\sigma_{x y} \frac{\partial W}{\partial y}\right]\right. \\
\left.+\frac{\partial}{\partial y}\left[\sigma_{y x} \frac{\partial W}{\partial x}+\sigma_{y y} \frac{\partial W}{\partial y}\right]\right)=-\mu W, \\
\frac{\partial \sigma_{x x}}{\partial x}+\frac{\partial \sigma_{x y}}{\partial y}=0, \frac{\partial \sigma_{y y}}{\partial y}+\frac{\partial \sigma_{y x}}{\partial x}=0 .
\end{gathered}
$$

Here $W(x, y)$ is the perpendicular deflection of the plate, and $\hat{\sigma}$ is the two-dimensional stress tensor. $\mu$ is the coefficient of the restoring force per unit area, and the bending moment of the plate is

$$
D=\frac{E h^{3}}{12\left(1-v^{2}\right)},
$$

where $E$ and $v$ are Young's modulus and Poisson's ratio of the plate.

Equations (1) and (2) are a system of nonlinear partial differential equations that has no general solution available in analytical form, so only approximate solutions for small values of $W$ can be sought.

Let $\hat{\sigma}^{0}$ be the stress tensor belonging to the evident solution of $W=0$. For infinitesimal $w$ deflections let $\hat{\sigma}=\hat{\sigma}^{0}+\delta \hat{\sigma}$, where $\delta \hat{\sigma}$ is also infinitesimal.

If $\hat{\sigma}$ is a solution to the equations, then (2) is satisfied, and only (1) has to be dealt with. As $\hat{\sigma}^{0}$ is constant in space, and $\sigma_{y x}^{0}=\sigma_{x y}^{0}=0$, with the notation of $\sigma_{x x}^{0}=\sigma_{y y}^{0}$ $=-P$ we get

$$
\begin{array}{r}
D \Delta^{2} w+h \frac{\partial^{2} w}{\partial x^{2}}\left(P+\delta \sigma_{x x}\right)+2 h \frac{\partial^{2} w}{\partial x \partial y} \delta \sigma_{x y}+h \frac{\partial^{2} w}{\partial y^{2}}\left(P+\delta \sigma_{y y}\right)-h\left[\frac{\partial w}{\partial x}\left[\frac{\partial \delta \sigma_{x x}}{\partial x}+\frac{\partial \delta \sigma_{x y}}{\partial y}\right]\right. \\
\left.+\frac{\partial w}{\partial y}\left[\frac{\partial \delta \sigma_{y x}}{\partial x}+\frac{\partial \delta \sigma_{y y}}{\partial y}\right]\right]=-\mu w
\end{array}
$$

As long as the derivatives of $w$ are finite, the terms containing $\delta \hat{\sigma}$ can be neglected, so we conclude to the following linearized equation:

$$
D \Delta^{2} w+h P \Delta w+\mu w=0 .
$$

As general solutions of the preceding equation are still not available, let us find the conditions of the existence of a periodic solution

$$
w=w_{0} \sin (k x), \quad k \neq 0,
$$

meaning the appearance of rippling in the $x$ direction. After substitution this leads to 


$$
D k^{4}-h P k^{2}+\mu=0,
$$

giving the relation between $P$ and $k$ if $\mu$ is known.

\section{Simplified version}

For an exactly rectangular stress profile, $\mu$ can be taken from Ref. 10 as

$$
\mu=\frac{2 E_{0} k}{\left(3-v_{0}\right)\left(1+v_{0}\right)}:=C k,
$$

where the 0 indices mark the elastic constants of the semispace. Thus periodic solutions with wave number $k$ appear at a plane stress of

$$
P=\frac{D k^{2}}{h}+\frac{C}{h k} .
$$

As $C, D, k$, and $h$ are all positive, (9) is of a parabolic nature; let its minimum be at $k=k_{\text {crit }}$. This means that after reaching the corresponding stress of $P_{\text {crit }}$, the plate will become instable against wrinkling with wave number $k_{\text {crit. }}$ The critical values are

$$
\begin{aligned}
& P_{\text {crit }}=\left(\frac{9 E E_{0}^{2}}{4\left(3-v_{0}\right)^{2}\left(1+v_{0}\right)^{2}\left(1-v^{2}\right)}\right)^{1 / 3}, \\
& k_{\text {crit }}=\left(\frac{12 E_{0}\left(1-v^{2}\right)}{E\left(3-v_{0}\right)\left(1+v_{0}\right)}\right)^{1 / 3} \frac{1}{h} .
\end{aligned}
$$

The value of $\mu$ used in the preceding formulas assumes an unstressed substrate and a plate (corresponding to the implanted layer) with a uniform stress across its thickness. Besides material constants, the wave number only depends on the plate thickness, which can be taken as a first approximation to be equal to $\Gamma$, the full width at half maximum of the implanted atom distribution. Formulas (10) $-(11)$ are not sensitive to the value of Poisson's ratio, taking into account that the latter can only be in the range $0-0.5$ in real materials. Thus setting $v=v_{0}=0.3$ does not introduce significant errors. Moreover, to get material independent approximations of the critical values, in the simplified version of the model we shall assume $E=E_{0}$, and get

$$
\begin{aligned}
& P_{\text {crit }}=0.58 E, \\
& \lambda=\frac{2 \pi}{k_{\text {crit }}}=4.3 \Gamma=10.2 \Delta R_{p},
\end{aligned}
$$

where $\Delta R_{p}$ is the ion range straggling (in the case of monoenergetic implants), and $\lambda$ is the predicted wavelength of the ripple structures formed by the elastic instability of the implanted layer.

\section{Realistic version}

As the simplified version is only an approximation in case of ion implantation, a more realistic picture is obtainable by keeping $\mu$ as a fitted parameter (i.e., allowing for the nonrectangular stress distribution). In this case the critical values from (7) are

$$
\begin{aligned}
& P_{\mathrm{crit}}^{*}=\left[\frac{\mu E h}{3\left(1-v^{2}\right)}\right)^{1 / 2}, \\
& k_{\mathrm{crit}}^{*}=\left(\frac{12 \mu\left(1-v^{2}\right)}{E}\right)^{1 / 4} \frac{1}{h^{3 / 4}} .
\end{aligned}
$$

\section{DISCUSSION}

Our model is valid only for ripples of infinitesimal amplitudes. However, if rippling starts with a well-defined $\lambda$, it can be justly expected that the wavelength will not change (significantly), while the amplitude grows during further ion bombardment.

In the simplified version the predicted value of $\lambda$ was obtained without using any fitted parameters. Despite this, it is in remarkable agreement with experimental data obtained in three kinds of experiments, i.e., after flaking, after excessive sputtering, or directly on the sample surface. ${ }^{11}$ However, the value of $P_{\text {crit }}$ obtained from the model in this form is rather large. This should be due to the assumed sharp interface between the stressed and unstressed media (rectangular distribution profile of implanted atoms).

In the more realistic version, when a fitted value of $\mu$ is used, the fit can be made separately for different groups of materials, to reintroduce the material dependence that was disregarded in the simplified version.

In Fig. 1 the solid line corresponds to formula (13), while the two dashed lines are fitted to the points obtained in experiments on either different kinds of metallic glasses or single-crystalline silicon. The points corresponding to high-dose sputtering experiments are also indicated.

Using the fitted value of the restoring coefficient $\mu$, (which is obviously smaller for real profiles), $P_{\text {crit }}^{*}$ is much smaller than $P_{\text {crit }}$ and it is comparable to stresses known to be attainable in high-dose ion-implantation experi-

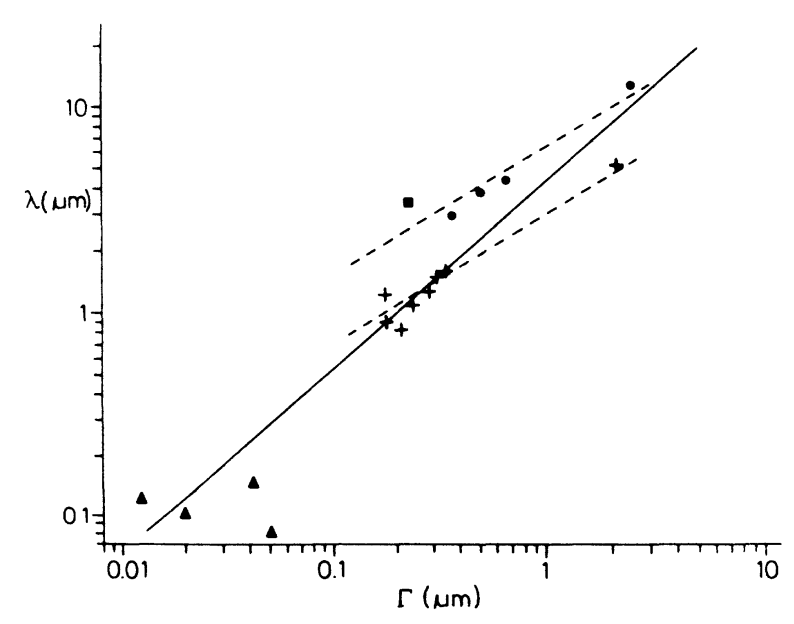

FIG. 1. The periodicity of wrinkling $(\lambda)$ vs the implanted atom distribution width $(\Gamma)$ observed in various experiments. $\Delta$ symbols denote the high dose sputtering experiments, the ones on single-crystal silicon, + the ones on metallic glasses, and the others. 
ments, e.g., Refs. 12 and 13.

Despite the vast amount of high-dose ion implantation experiments carried out on various polycrystalline samples, we know of only one instance, where similar ripple patterns were observed. ${ }^{2}$ We ourselves did not observe the phenomenon on the polycrystalline modification of the very same metallic glass that did exhibit ripple formation in its amorphous state. ${ }^{14}$ This is in accord with the implicit assumption of the model, i.e., that the sample is crystallographically homogeneous in a region large enough to accommodate a reasonable number of ripple trains. If this is not the case like, in polycrystalline targets, the uniform and large enough stress field cannot build up to cause elastic instability. In the case mentioned in Ref. 2 , the pattern was only seen on a single grain of the sample, which was a large enough singlecrystalline region for the phenomenon to occur.

Another necessary condition for the appearance of wrinkling is the attainment of the critical stress. Of course this does not necessarily happen in all cases, no matter how high the implanted dose is. For example, other surface deformation processes (flaking, blistering, exfoliation) may take place before the critical stress is reached.

The direction of the ripples is expected to be perpendicular to the direction of largest stress. In symmetric cases this direction is selected by minor imperfections, but it can also be determined externally. Such an experiment has already been conducted, ${ }^{14}$ when two metallic glass ribbons of about $50-\mu \mathrm{m}$ thickness were implanted together along their edges. As expected, the direction of the ripples was transversal, i.e., perpendicular to the direction of larger stress.

Thus the model is in remarkable agreement with the available experimental data. There were, however, no experiments conducted with changing only the control parameter, i.e., the width of the implanted atom distribution. This gave rise to speculations that the ripple wavelength may depend not on the range straggling, but on the projected range in case of monoenergetic implantation. Experiments that exclude this assumption are reported in the following paper. ${ }^{15}$

\section{CONCLUSION}

The stress model for the wrinkling of ion-implanted layers has been applied successfully for experiments producing ripple morphologies under various conditions of high-dose ion bombardment, ${ }^{1-6}$ Fig. 1. The correlation between the wavelength of rippling and the width of the implanted-atom distribution is noticed for the first time, and an explanation is given for the formation of the surface morphologies.
${ }^{1}$ G. Carter, B. Navinsek, and J. L. Whitton, in Sputtering by Particle Bombardment II, edited by R. Behrisch (SpringerVerlag, Berlin, 1983), p. 253.

${ }^{2}$ D. K. Sood et al., J. Nucl. Mater. 79, 423 (1979).

${ }^{3}$ A. Manuaba et al., Nucl. Instrum. Methods 199, 400 (1982).

${ }^{4}$ Y. Watanabe, S. Nanao, and A. Kohyama, J. Nucl. Mater. 122\&123, 743 (1984).

${ }^{5}$ F. Pászti et al., Nucl. Instrum. Methods B7/8, 371 (1985).

${ }^{6}$ G. Carter, M. J. Nobes, and J. L. Whitton, Appl. Phys. A38, 77 (1985).

${ }^{7}$ I. Lovas and T. Tél, Phys. Rev. B 28, 5692 (1983).

${ }^{8}$ R. M. Bradley and J. M. E. Harper, J. Vac. Sci. Technol. A6,
2390 (1988).

${ }^{9}$ L. D. Landau and E. M. Lifschitz, Elastizitäts-theorie (Akademie Verlag, Berlin, 1970), p. 64.

${ }^{10}$ H. G. Allen, Analysis and Design of Structural Sandwich Panels (Pergamon, Oxford, 1969), p. 157.

${ }^{11}$ C. Hajdu, F. Pászti, M. Fried, and I. Lovas, Nucl. Instrum. Methods B19/20, 607 (1987).

${ }^{12}$ E. P. EerNisse and S. T. Picraux, J. Appl. Phys. 48, 9 (1977).

${ }^{13}$ N. E. W. Hartley, J. Vac. Sci. Technol. 12, 485 (1975).

${ }^{14}$ F. Pászti et al., Nucl. Instrum. Methods 209/210, 273 (1983).

${ }^{15}$ M. Fried et al., following paper, Phys. Rev. B 41, 3923 (1990). 